\title{
Two exactly-solvable problems in one-dimensional hyperbolic Space
}

\author{
Č. Burdík ${ }^{1,2}$ and G.S.Pogosyan ${ }^{1,3}$ \\ ${ }^{1}$ Laboratory of Theoretical Physics, Joint Institute for Nuclear Research, \\ Dubna, 141980, Moscow Region, Russia \\ 2 Department of Mathematics Czech Technical University, Trojanova 13, 120 00, \\ Prague, Czech Republic \\ ${ }^{3}$ Centro de Ciencias Físicas UNAM, Apartado Postal 48-3 \\ 62251 Cuernavaca, Morelos, México
}

\begin{abstract}
In this paper we establish a relation between two exactly-solvable problems on one-dimensional hyperbolics space, namely singular Coulomb and singular oscillator systems.
\end{abstract}

1. In the last few years the relation between Coulomb and oscillator problems on the Ndimensional spaces with constant curvature (both positive on the sphere $S_{n}$ and negative on hyperboloid $H_{n}$ ) and complex projective spaces $C P^{N}$ have been discussed in many papers [1]-[5]. The first investigation for one spatial dimension was done in the two recent papers $[6,7]$. In particular in the article [6] have constructed the complex mapping $S_{1 C} \rightarrow S_{1}$, which extend in spherical geometry well-known for Euclidean space Hurwitz transformation. It was also shown that this transformation establish the correspondence between Coulomb and singular oscillator systems in one dimensions.

In the present note we find the relation between two exactly-solvable potentials on one-dimensional hyperbolic space $H_{1}: s_{0}^{2}-s_{1}^{2}=R^{2}$

$$
V^{s o}(\vec{s})=\frac{\omega^{2} R^{2}}{2} \frac{s_{1}^{2}}{s_{0}^{2}}+\frac{1}{2} \frac{k^{2}-\frac{1}{4}}{s_{1}^{2}}, \quad V^{s c}(\vec{s})=-\frac{\mu}{R} \frac{s_{0}}{\left|s_{1}\right|}+\frac{1}{2} \frac{p^{2}-\frac{1}{4}}{s_{1}^{2}},
$$

where $s_{0}, s_{1}$ are Cartesian coordinates in the ambient pseudo-euclidean space $E_{1,1}$ and $k$, and $p$ are positive.

The potentials in (1) belong to the well-known class of superintegrable potentials in the constant curvature spaces $[8,9]$ restricted to one spatial dimension. The ordinary 1D oscillator system $(k=1 / 2)$ is singular at the point $s_{0}=0$, which separate the motion on the upper and lower sheets of hyperbola. The ordinary $1 \mathrm{D}$ Coulomb system $(p=1 / 2)$ is attractive on upper and repulsive on lower sheets of hyperbola. Below we will consider only the motion on the upper sheet of hyperbola $\left(s_{0}>0\right)$. 
2. The Schrödinger equation describing the nonrelativistic quantum motion on onedimensional hyperbolic space $H_{1}$ in pseudospherical coordinates $\tau \in(-\infty, \infty)$

$$
s_{0}=R \cosh \tau, \quad s_{1}=R \sinh \tau,
$$

has the following form $(\hbar=\mu=1)$

$$
\frac{d^{2} \Psi}{d \tau^{2}}+2 R^{2}[E-V(\tau)] \Psi=0
$$

Let us consider first the eq. (3) for potential $V(\tau)=V^{s o}(\tau)$. Taking into account that in pseudospherical coordinates $(2)$

$$
V^{s o}(\tau)=\frac{\omega^{2} R^{2}}{2} \frac{s_{1}^{2}}{s_{0}^{2}}+\frac{1}{2} \frac{k^{2}-\frac{1}{4}}{s_{1}^{2}}=\frac{1}{2 R^{2}}\left(-\frac{k_{0}^{2}-\frac{1}{4}}{\cosh ^{2} \tau}+\frac{k^{2}-\frac{1}{4}}{\sinh ^{2} \tau}\right)+\frac{\omega^{2} R^{2}}{2},
$$

where $k_{0}^{2}=\omega^{2} R^{4}+\frac{1}{4}$ we obtain from eq.(3)

$$
\frac{d^{2} \Psi}{d \tau^{2}}+\left[\epsilon+\frac{k_{0}^{2}-\frac{1}{4}}{\cosh ^{2} \tau}-\frac{k^{2}-\frac{1}{4}}{\sinh ^{2} \tau}\right] \Psi=0
$$

with $\epsilon=2 R^{2} E-\omega^{2} R^{4}$. Thus the singular oscillator problem on the hyperbola $H_{1}$ is described by the well-known modified Pöschl-Teller equation and, unlike the Schrödinger equation for $1 \mathrm{D}$ singular oscillator on the flat space $E_{1}$ and the sphere $S_{1}$, the eq. (5) possesses both discrete $(\epsilon<0)$ and continuous $(\epsilon \geq 0)$ spectrum.

The bound state wave functions is given by $[8,9]$

$$
\Psi_{n}^{\left( \pm k, k_{0}\right)}(\tau)=N_{n}^{\left( \pm k, k_{0}\right)}(\sinh \tau)^{\frac{1}{2} \pm k}(\cosh \tau)^{2 n-k_{0}+\frac{1}{2}}{ }_{2} F_{1}\left(-n, k_{0}-n ; 1 \pm k ; \tanh ^{2} \tau\right)
$$

where $N_{n}^{\left( \pm k, k_{0}\right)}$ is the normalization constant. The $\epsilon$ is quantized as

$$
\epsilon=-\left(2 n+1 \pm k-k_{0}\right)^{2}
$$

with $n=0,1, \ldots\left[\frac{1}{2}\left(k_{0} \mp k-1\right)\right]$ and a bound state solution is possible only for $k_{0} \mp k>1$. Then the discrete energy spectrum takes the value

$$
E_{n}^{ \pm k}(R)=-\frac{1}{2 R^{2}}\left[(2 n+1 \pm k)^{2}-2 k_{0}(2 n+1 \pm k)+\frac{1}{4}\right] .
$$

The wave functions $\Psi_{n}^{\left( \pm k, k_{0}\right)}(\tau)$ satisfies the orthogonality relation

$$
R \int_{0}^{\infty} \Psi_{n}^{\left( \pm k, k_{0}\right)}(\tau) \Psi_{n^{\prime}}^{\left( \pm k, k_{0}\right)}(\tau) d \tau=\frac{1}{2} \delta_{n n^{\prime}}
$$

and the normalization factor has the form

$$
N_{n}^{\left( \pm k, k_{0}\right)}(R)=\frac{1}{\Gamma(1 \pm k)} \sqrt{\frac{\left(-2 n+k_{0} \mp k-1\right) \Gamma\left(-n+k_{0}\right) \Gamma(n+1 \pm k)}{R \Gamma\left(-n+k_{0} \mp k\right) n !}}
$$


The positive sign at the $k$ has to taken whenever $k>\frac{1}{2}$, i.e. the additional term to oscillator potential is repulsive at the origin and the motion take place only in one of the domains $\tau \in[0, \infty)$ or $\tau \in[0,-\infty)$.

If $0<k \leq \frac{1}{2}$, i.e. the additional term is attractive at the origin, the motion take place in $\tau \in(-\infty, \infty)$ and both the positive and negative sign must be taken into account in the solution. Thus there exists two family of solutions go over into each other under the change $k \rightarrow-k$. This is indicated by the notion $\pm k$, in the formulas.

In the contraction limit $R \rightarrow \infty, \tau \rightarrow 0, R \tau \sim x$ - fixed and $k_{0} \sim \omega R^{2}$ we see that the continuous spectrum is vanishing while the discrete spectrum is infinite. It is easy to reproduce the energy spectrum

$$
E_{n}^{ \pm k}=\lim _{R \rightarrow \infty} E_{n}^{ \pm k}(R)=\omega(2 n+1 \pm k)
$$

and the wave function

$$
\Psi_{n}^{ \pm k}(x)=\lim _{\substack{R \rightarrow \infty \\ \tau \rightarrow 0}} \Psi_{n}^{\left( \pm k, k_{0}\right)}(\tau)=\sqrt{\frac{\sqrt{\omega} \Gamma(n+1 \pm k)}{n ![\Gamma(1 \pm k)]^{2}}}(\sqrt{\omega} x)^{\frac{1}{2} \pm k} e^{-\frac{\omega x^{2}}{2}}{ }_{1} F_{1}\left(-n ; 1 \pm k ; \omega x^{2}\right),
$$

for the flat 1D singular oscillator system [10].

3. The 1D singular Coulomb potential in the pseudospherical coordinates (2) has the form

$$
V^{s c}(\tau)=-\frac{\mu}{R}\left(\frac{s_{0}}{\left|s_{1}\right|}-1\right)+\frac{1}{2} \frac{p^{2}-\frac{1}{4}}{s_{1}^{2}}=-\frac{\mu}{R}(\operatorname{coth}|\tau|-1)+\frac{1}{2 R^{2}} \frac{p^{2}-\frac{1}{4}}{\sinh ^{2} \tau} .
$$

Let us first consider the region $\tau \geq 0$. Substituting the potential (12) in eq.(3), we arrive to equation

$$
\frac{d^{2} \Psi}{d \tau^{2}}+\left[\left(2 R^{2} E-2 \mu R\right)+2 \mu R \operatorname{coth} \tau-\frac{p^{2}-\frac{1}{4}}{\sinh ^{2} \tau}\right] \Psi=0
$$

which is known as the Manning-Rosen potential problem [11]. Making now the transformation from variable $\tau$ to the new variable $\alpha \in[0, \infty)$

$$
e^{\tau}=\cosh \alpha
$$

and setting $\Psi(\alpha)=W(\alpha) / \sqrt{\operatorname{coth} \alpha}$. As result we obtain the equation

$$
\frac{d^{2} W}{d \alpha^{2}}+\left[2 R^{2} E+\frac{\left(-2 R^{2} E+4 \mu R\right)-\frac{1}{4}}{\cosh ^{2} \alpha}-\frac{4 p^{2}-\frac{1}{4}}{\sinh ^{2} \alpha}\right] W=0
$$

It is easy to see that, up to the substitution

$$
\epsilon=2 R^{2} E, \quad k_{0}^{2}=-2 R^{2} E+4 \mu R, \quad k^{2}=4 p^{2} .
$$


the equation (15) for the 1D singular Coulomb problem coincides with the 1D singular oscillator equation (5). The regular for $\alpha \in[0, \infty)$ solution of this equation according to (6) is

$$
W_{n}(\alpha)=A_{n}(\sinh \alpha)^{\frac{1}{2} \pm k}(\cosh \alpha)^{2 n-k_{0}+\frac{1}{2}}{ }_{2} F_{1}\left(-n,-n+k_{0} ; 1 \pm k ; \tanh ^{2} \alpha\right)
$$

where $A_{n}$ is a normalization constant. The constant $A_{n}$ is computed from the requirement that the wave function (17) satisfies the normalized condition

$$
R \int_{0}^{\infty}\left|\Psi_{n}(\tau)\right|^{2} d \tau=R \int_{0}^{\infty}\left|W_{n}(\alpha)\right|^{2} \tanh ^{2} \alpha d \alpha=\frac{1}{2},
$$

and has the following form

$$
A_{n}=\sqrt{\frac{k_{0}\left(k_{0}-2 n \mp k-1\right) \Gamma\left(k_{0}-n\right) \Gamma(n+1 \pm k)}{R(2 n+1 \pm k)(n) ![\Gamma(1 \pm k)]^{2} \Gamma\left(k_{0}-n \mp k\right)}} .
$$

Let us now consider the two most interesting cases.

3.1 In the case when $p=\frac{1}{2}$ the centrifugal term in $V^{s c}$ disappears and the motion take place in the region $\tau \in(-\infty, \infty)$. Then the transformation (14) establishes the connection between ordinary 1D Coulomb problem and 1D singular oscillator with $k=1$. Comparing now eq.(16) with (7), we get

$$
k_{0}=(n+1+\sigma), \quad \sigma=\frac{\mu R}{n+1} .
$$

and for the discrete energy spectrum

$$
E_{n}(R)=-\frac{(n+1)^{2}}{2 R^{2}}-\frac{\mu^{2}}{2(n+1)^{2}}+\frac{\mu}{R}, \quad n=0,1,2, \ldots[\sigma-1]
$$

Returning to the variable $\tau$, we find for bound state wave function $(\tau>0)$

$$
\Psi_{n \sigma}(\tau)=A_{n}(\sigma) \sinh \tau e_{2}^{(n-\sigma) \tau} F_{1}\left(-n, 1+\sigma ; 2 ; 1-e^{-2 \tau}\right)
$$

where the normalization constant $A_{n}(\sigma)$ is

$$
A_{n}(\sigma)=\sqrt{\frac{2 \sigma\left[\sigma^{2}-(n+1)^{2}\right]}{R}}
$$

In the domain $-\infty<\tau<0$ 1D Coulomb wave function are determined from eq. (22) after reflection $\tau \rightarrow-\tau$. Finally the general solution of the Schrödinger equation for $-\infty<\tau<\infty$ then can be presented in form of even and odd functions

$$
\begin{aligned}
& \Psi_{n \sigma}^{(+)}(\tau)=A_{n}(\sigma) \sinh |\tau| e^{(n-\sigma)|\tau|}{ }_{2} F_{1}\left(-n, 1+\sigma ; 2 ; 1-e^{-2|\tau|}\right) \\
& \Psi_{n \sigma}^{(-)}(\tau)=A_{n}(\sigma) \sinh \tau e^{(n-\sigma)|\tau|}{ }_{2} F_{1}\left(-n, 1+\sigma ; 2 ; 1-e^{-2|\tau|}\right)
\end{aligned}
$$


Thus we have seen that using the transformation (14) we can completely solve 1D Coulomb system on hyperbola $H_{1}$, including eigenfunctions and discrete energy spectrum.

Now let us consider the flat space contraction. In the contraction limit $R \rightarrow \infty$ the energy spectrum for finite $n$ goes to the energy spectrum of the 1D hydrogen atom [12]

$$
E_{n}=\lim _{R \rightarrow \infty} E_{n}^{\nu}(R)=-\frac{\mu^{2}}{2(n+1)^{2}}, \quad n=0,1,2, \ldots
$$

In the limit $R \rightarrow \infty$, putting $\tau \sim x / R$ in eqs. (24) and (25), we obtain (up to the factor $\sqrt{2}$ ) the well-known 1D Coulomb wave function [12]

$$
\begin{aligned}
& \lim _{\substack{R \rightarrow \infty \\
\tau \rightarrow 0}} \Psi_{n \sigma}^{(+)}(\varphi)=\sqrt{\frac{2 \mu^{3}}{(n+1)^{3}}}|x| e^{-\frac{\mu|x|}{n}}{ }_{1} F_{1}\left(-n ; 2 ; \frac{2 \mu|x|}{n}\right), \\
& \lim _{\substack{R \rightarrow \infty \\
\tau \rightarrow 0}} \Psi_{n \sigma}^{(-)}(\varphi)=\sqrt{\frac{2 \mu^{3}}{(n+1)^{3}}} x e^{-\frac{\mu|x|}{n}}{ }_{1} F_{1}\left(-n ; 2 ; \frac{2 \mu|x|}{n}\right) .
\end{aligned}
$$

3.2. Let us now choose $k=\frac{1}{2}^{-}$. Then $p^{2}-\frac{1}{4}=-3 / 16$ and the centrifugal term is attractive at the origin for potential $V^{s c}$. As in the previous case the motion take place in the domain $-\infty<\tau<\infty$. For potential $V^{s o}$ this term vanish and therefore transformation (14) connect the ordinary oscillator with 1D singular Coulomb system.

Let us now introduce the quantity $\nu=\frac{1}{2}(1 \pm k)$, which takes two values $\nu=\frac{1}{4}$ and $\nu=\frac{3}{4}$. Making the all calculation by analogy to previous case, it easy to get the discrete energy spectrum

$$
E_{n}^{\nu}(R)=-\frac{(n+\nu)^{2}}{2 R^{2}}-\frac{\mu^{2}}{2(n+\nu)^{2}}+\frac{\mu}{R}, \quad n=0,1,2, \ldots
$$

and normalized bound state wave function

$$
\begin{aligned}
\Psi_{n \sigma_{\nu}}^{\nu}(\tau) & =2^{\nu} \sqrt{\frac{\left[\sigma_{\nu}^{2}-(n+\nu)^{2}\right] \Gamma\left(\sigma_{\nu}+\nu\right) \Gamma(n+2 \nu)}{2 R(n+\nu) n ! \Gamma\left(\sigma_{\nu}+1-\nu\right)[\Gamma(2 \nu)]^{2}}} . \\
& \times(\sinh \tau)^{\nu} e^{\tau\left(n-\sigma_{\nu}\right)}{ }_{2} F_{1}\left(-n, \nu+\sigma_{\nu} ; 2 \nu ; 1-e^{-2 \tau}\right),
\end{aligned}
$$

where $\sigma_{\nu}=\mu R /(n+\nu)$.

In the flat space limit $R \rightarrow \infty$, it is easy to see

$$
E_{n}^{\nu}=\lim _{R \rightarrow \infty} E_{n}^{\nu}(R)=-\frac{\mu^{2}}{2(n+\nu)^{2}}, \quad n=0,1,2, \ldots
$$

and

$$
\Psi_{n}^{\nu}(y)=\lim _{\substack{R \rightarrow \infty \\ \tau \rightarrow 0}} \Psi_{n \sigma}^{\nu}(\varphi)=\frac{\sqrt{\mu}}{\Gamma(2 \nu)} \frac{1}{(n+\nu)} \sqrt{\frac{\Gamma(n+2 \nu)}{2 n !}} y^{\nu} e^{-\frac{y}{2}}{ }_{1} F_{1}(-n ; 2 \nu ; y),
$$

where $y=2 \mu x /(n+\nu)$. Formulas (29) and (30) coincides with the formulas for energy levels $E_{n}^{\nu}$ and up to the factor $\sqrt{2}$ for wave functions $\Psi_{n}^{\nu}(y)$ for the two type of onedimensional Coulomb anyons with $\nu=\frac{1}{4}$ and $\nu=\frac{3}{4}$ respectively [13]. 


\section{References}

[1] E.G.Kalnins, W.Miller Jr. and G.S.Pogosyan. J.Math.Phys. 41, 2629-2657, 2000.

[2] A.Nersessian and G.S.Pogosyan. Phys.Rev A63, 20103 (R), 2000; [ArXiv: quant$\mathrm{ph} / 0006118]$.

[3] A.Nersessian. Physics of Atomic Nuclei, 65, 1103-1108, 2002

[4] E.G.Kalnins, W.Miller Jr. and G.S.Pogosyan. Physics of Atomic Nuclei, 65(6), 10861094, 2002.

[5] S.Bellucci and A.Nersessian. Phys. Rev. D67, 065013, 2003. [ArXiv: hep-th/0211070]

[6] L.G.Mardoyan, G.S.Pogosyan and A.N.Sissakian. Theoretical and Mathematical Physics, 135(3), 808-813, 2003

[7] L.G.Mardoyan, G.S.Pogosyan and A.N.Sissakian. [ArXiv: quant-ph/0303016].

[8] C.Grosche, G.S.Pogosyan and A.N.Sissakian. Fortschritte der Physik, 43(6), 523563, 1995. Phys. Part. Nucl. 27(3), 244-278, 1996; 28, 486-519, 1997.

[9] E.G.Kalnins, W.Miller Jr. and G.S.Pogosyan. J.Math.Phys. 38, 5416-5433, 1997.

[10] Ye.M.Hakobyan, M.Kibler, G.S.Pogosyan and A.N.Sissakian. Physics of Atomic Nuclei, 61(10), 1782-1788, 1998.

[11] M.F.Manning, N.Rosen. Phys. Rev. 44, 953, 1933.

[12] I.V. Lutsenko, L.G. Mardoyan, G.S. Pogosyan, A.N. Sissakian, V.M. Ter-Antonyan. J.Phys. A22, 2739, 1989.

[13] V.M.Ter-Antonyan. Dyon -Oscillator Duality, quant-ph/0003106, 2000. 